\title{
Infectomic Analysis of Gene Expression Profiles of Human Brain Microvascular Endothelial Cells Infected with Cryptococcus neoformans
}

\author{
Ambrose Jong, ${ }^{1}$ Chun-Hua Wu, ${ }^{1}$ Wensheng Zhou, ${ }^{2}$ Han-Min Chen, ${ }^{3}$ and Sheng-He Huang ${ }^{1}$ \\ ${ }^{1}$ Divisions of Hematology-Oncology and Infectious Diseases, Saban Research Institute of Childrens Hospital Los Angeles, \\ Keck School of Medicine, University of Southern California, Los Angeles, CA 90027, USA \\ ${ }^{2}$ HRL Laboratories, LLC, Malibu, CA 90265, USA \\ ${ }^{3}$ Department of Life Science, Fu Jen Catholic University, Hsinchuang, Taipei Country 24205, Taiwan
}

Correspondence should be addressed to Ambrose Jong, ajong@chla.usc.edu

Received 12 August 2007; Accepted 17 December 2007

Recommended by Daniel Howard

In order to dissect the pathogenesis of Cryptococcus neoformans meningoencephalitis, a genomic survey of the changes in gene expression of human brain microvascular endothelial cells infected by $C$. neoformans was carried out in a time-course study. Principal component analysis (PCA) revealed significant fluctuations in the expression levels of different groups of genes during the pathogen-host interaction. Self-organizing map (SOM) analysis revealed that most genes were up- or downregulated 2 folds or more at least at one time point during the pathogen-host engagement. The microarray data were validated by Western blot analysis of a group of genes, including $\beta$-actin, Bcl-x, CD47, Bax, Bad, and Bcl-2. Hierarchical cluster profile showed that 61 out of 66 listed interferon genes were changed at least at one time point. Similarly, the active responses in expression of MHC genes were detected at all stages of the interaction. Taken together, our infectomic approaches suggest that the host cells significantly change the gene profiles and also actively participate in immunoregulations of the central nervous system (CNS) during C. neoformans infection.

Copyright (c) 2008 Ambrose Jong et al. This is an open access article distributed under the Creative Commons Attribution License, which permits unrestricted use, distribution, and reproduction in any medium, provided the original work is properly cited.

\section{INTRODUCTION}

The major challenge posed by infectious diseases is to holistically and integratively understand the fundamental issues of how infectious agents and human hosts interact during microbial infection.Host-microbe interactions in the pathogenesis of infectious diseases are dynamic and complex processes [1] which result in changes in whole genome expression profiles of microbial pathogens and their hosts. A new discipline called infectomics [1] became established when the recently developed high-throughput omic approaches and computational tools were combined with the conventional approaches for the study of infectious diseases. Infectomes are detailed maps of microbial infections, and the availability of whole genomes of many living organisms paves the way for their holistic and integrative study. Infectomics can be defined as the study of infectomes, which are encoded by genomes of microbes and their hosts. Microarray has been a powerful tool to monitor infectomes in microorganisms and their host responses during microbial infection.

Infection by Cryptococcus neoformans has increased considerably over the past few years [2-4]. Dehydrated haploid yeast or basidiospore of $C$. neoformans is the usual form of inhalation $[3,5]$. The organisms are likely to spread hematogeneously to extrapulmonary tissues and show a remarkable propensity in spreading to the brain and meninges, where life-threatening meningoencephalitis develops $[2,6,7]$. In order to cause meningoencephalitis, $C$. neoformans must penetrate the blood-brain barrier (BBB), which is a barrier between blood circulation and the brain parenchyma. BBB mainly consists of brain microvascular endothelial cells (BMECs), which are responsible for maintaining the biochemical homeostasis within the central nervous system (CNS) [8-10]. BMEC has been established as an in vitro cell culture model for dissecting the underlying mechanism(s) whereby $C$. neoformans crosses the BBB. We have recently 
demonstrated that $C$. neoformans are able to alter the cytoskeleton of human brain microvascular endothelial cells (HBMECs) [11]. We have also identified and characterized a C. neoformans capsule gene, CPS1 [12]. This demonstrated that CPS1 encodes hyaluronic acid synthase. The above information suggested that $C$. neoformans hyaluronic acid (HA) plays a role as an adhesion molecule during the yeast entry. It also suggested that host cell factors are required for $C$. neoformans HA-binding and the pathogen entry into HBMEC [12].

Like many other pathogens, C. neoformans may manipulate the host system to facilitate its invasion. The investigation of virulence of the pathogen C. neoformans and the study of the responses from HBMEC are equally important in the understanding of the complex invasion process. A more comprehensive knowledge of the interplay between the host and microbial pathogen at the levels of genome expression profiles is central to the understanding of the pathogenesis of infectious diseases. In order to dissect the pathogenesis of this disease, we have combined the infectomic approach with the in vitro model of the BBB to monitor gene expression profiles of HBMECs infected with $C$. neoformans. These studies provide global and useful information for building a comprehensive framework to interpret C. neoformans pathogenic processes.

\section{MATERIALS AND METHODS}

\subsection{Cultures of yeasts and human brain microvascular endothelial cells (HBMECs)}

C. neoformans strain B3501 was used for this study [11]. Yeast cells were grown aerobically at $30^{\circ} \mathrm{C}$ in the rich YPD broth containing $1 \%$ yeast extracts, $2 \%$ peptone, and $2 \%$ dextrose. Cells were harvested at early log phase, washed with PBS, and resuspended in Ham-F12/M199 (1:1; v:v) and 5\% heat inactivated fetal bovine serum (FBS) (experimental medium).

The HBMEC culture was prepared as described previously $[11,13]$. Briefly, the HBMEC cultures were maintained in RPMI 1640 medium including 10\% FBS and 10\% NuSerum (BD Biosciences, Bedford, MA, USA) at $37^{\circ} \mathrm{C}$ in a humid atmosphere of $5 \% \mathrm{CO}_{2}$ as described above. For the preparation of interactive cultures for microarray analysis, the HBMEC were grown in collagen-coated 24 well tissue culture plates (Costar Corp, Cambridge, MA, USA) until confluency. An inoculum of $10^{6}$ yeast cells in $1 \mathrm{~mL}$ experimental medium was added. C. neoformans B3501 was incubated with $\mathrm{HBMEC}$ at $37^{\circ} \mathrm{C}$ and harvested at $0,4,8,12,16$, 20, 24 hours. One-tenth of cell pellets were saved for Western blots and the rest were subjected to the RNA extraction for making the probes.

\subsection{Preparation of the biotinylated CRNA for microarray}

Total RNA was preparedusing TRIZOL reagent (Invitrogen, Calif, USA), and subjectedto isolation of poly $(\mathrm{A})^{+} \mathrm{RNA}$ using the Oligotex-dT30 mRNA purification kit (TaKaRa Shuzo Co., Kyoto, Japan) according to the manufacturer's instructions. The biotinylated cRNA probe was prepared using the RNA Transcript labeling kit (Enzo Biochem, Farming- dale, NY, USA) according to the manufacturer's instructions. The quality of the probe was first examined with $1 \%$ agarose gel, showing the bands between $0.5 \sim 1.5 \mathrm{~kb}$, peaking at around $0.75 \mathrm{~kb}$. An aliquot of biotinylated cRNA probes was then examined by the Affymetirx test chips. Internal controls, G3PDH and $\beta$-actin, on the test chip were measured to evaluate the biotinylated probes. Only high-quality cRNA probes were used to hybridize with DNA microarray HU95A chips $\left(12,809\right.$ gene spots in $\sim 1.64 \mathrm{~cm}^{2}$ filters $)$. After hybridization, the HP GeneArray scanner was used to analyze the patterns of gene expression.

\subsection{Microarray analysis}

Biotinylated cRNA probes were prepared from $10 \mu \mathrm{g}$ of poly $(\mathrm{A})^{+}$RNA. Hybridization and fluorescence detection were performed according to the manufacturer's instructions. Images were analyzed with GeneSpring, Genetrix softwares. Three clustering approaches were used in this study as follows. (a) Hierarchical clustering where the data points were organized in a phylogenetic tree in which the branch lengths represent the degree of similarity between the values. (b) Self-organizing maps (SOMs) that was a nonhierarchical clustering approach. Using this algorithm, gene expression data were transformed into vectors or coordinates in an $n$-dimensional space, where $n$ equals the number of variables or time points. (c) Principal-component analysis (PCA) that was used to obtain a simplified visualization of entire datasets.

\subsection{Western blot analysis}

Protein concentration was determined by Bio-Rad protein assay and equal amounts of protein were used from different time point samples. SD $\widetilde{S}$-PAGE sample buffer $(50 \mathrm{mM}$ Tris- $\mathrm{HCl}$ pH 6.8, 10\% $\beta$-mercaptoethanol, $2 \%$ SD $\widetilde{S}, 0.1 \%$ bromophenol blue, $10 \%$ glycerol) was added to the samples and they were boiled in a water bath for $10 \mathrm{~min}$ utes. $2 \mu \mathrm{g}$ of total cell extracts were separated on homogeneous 12.5\% PhastGel (Phastsystem, Amersham Pharmacia Biotech, NJ, USA) with SDS buffer strips according to manufacturers instructions with subsequent transfer to PVDF membrane for 40 minutes. The membrane was blocked by $0.5 \%$ blocking solution (skim milk-based), incubated with anti-Bcl-2 antibody, anti-Bad antibody, anti-Bcl-x antibody, anti-Bax antibody (Transduction Laboratories, Calif, USA), anti-CD47 antibody (Lab Vision, Calif, USA), or anti- $\beta$-actin antibody (Chemicon Interna-tional, Calif) at $25^{\circ} \mathrm{C}$ followed by incubation with peroxidase-coupled secondary antibody (Kirkegaard Perry Laboratories, Md, USA) and detected by the ECL-enhanced chemiluminescent system (Boehringer Mannheim, Germany).

\section{RESULTS}

\subsection{Kinetics of gene expression of HBMEC showing the progression of gene profile changes during C. neoformans infection}

Microbial invasion is a complex and dynamic process. We performed a time-course study to examine the gene 


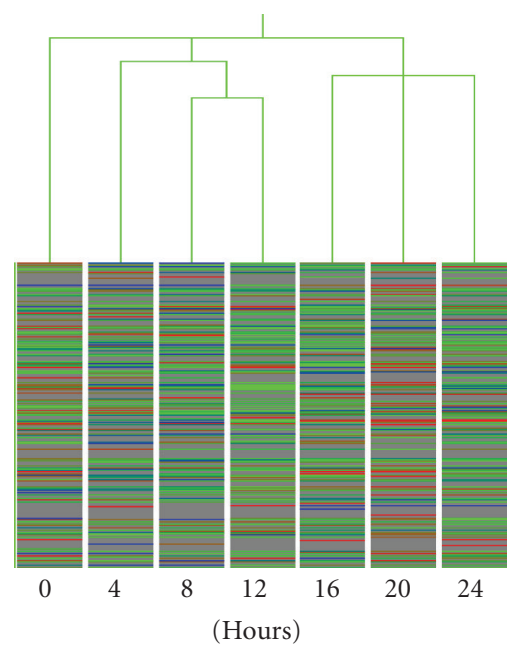

FIGURE 1: Gene expression profiles during C. neoformans infection; microarray analysis of mRNA levels was assessed at $0,4,8,12,16$, 20 , and 24 hours after $C$. neoformans incubation. Time points are represented by columns, and genes in rows. Red, green, and blue represent the higher, equal, and lower mRNA level relative to that of zero time point. A hierarchical clustering analysis of genes with expression levels that changed during C. neoformans and HBMEC interactions was performed. The distance among infectomic profiles of HBMEC is shown. Each lane represents a result form a gene chip. Genes with high intensity reading are shown in bright colors; genes with low intensity reading are in dim colors (grey) so they can be distinguished during analysis.

expression profiles in HBMEC during C. neoformans infection. We selected C. neoformans strain B3501 for this study because the genomic sequence of this strain has been completed. In addition, we have used this strain for in vitro adhesion and transcytosis studies on HBMEC $[11,13]$. It is an encapsulated strain with moderate adhesion activity to HBMEC, which was used as the in vitro model of BBB. A spectrum of 24 hours would allow us to evaluate its effect on HBMEC. Poly $(\mathrm{A})^{+}$RNAs derived from the incubated HBMEC at $0,4,8,12,16,20$, or 24 hours after B3501 incubation were subjected to the RNA extraction and the preparation of the biotinylated cRNA probe. The prepared probes at different time points were hybridized with biochip, individually. We used an Affymetric HU95A microarray chip harboring 12,559 human clones, facilitating efficient detection of changes in gene expression in the host cells. The expression levels were measured and analyzed by the GeneChip program. In a hierarchical clustering, the closet pair of expression values is grouped and the data points are organized in a phylogenetic tree in which the branch lengths represent the degree of similarity between the values. The mRNA profiles of HBMEC, assessed at 0, 4, 8, 12, 16, 20, and 24 hours after C. neoformans incubation, are shown in Figure 1.

These 12,559 gene patterns were classified into two major clusters (Clusters $0-12$ hours and 16-24 hours) by using the GeneSpring software. Rapid changes in gene profiles at an early time point are followed by a gradual alteration until the 12-hour time point. Of interest, the gene patterns of

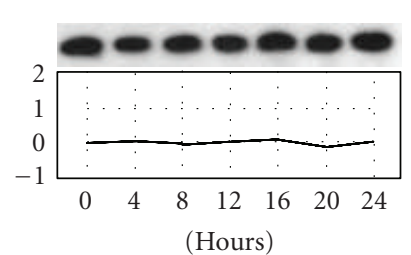

(a)

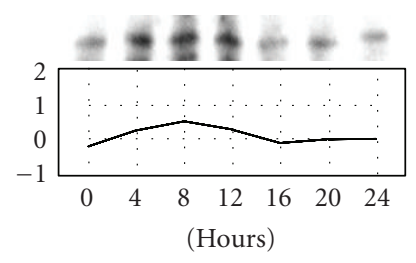

(c)

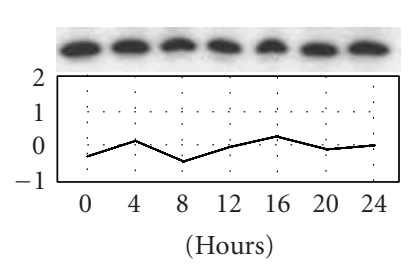

(e)

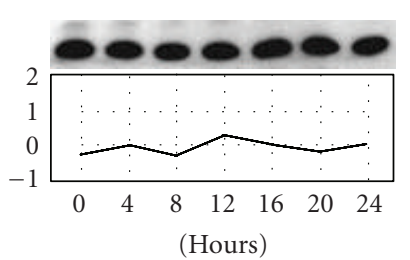

(b)

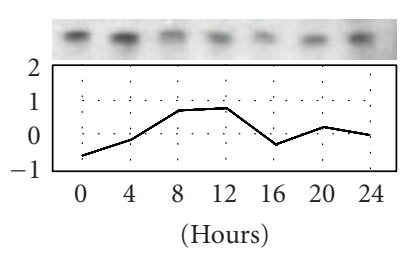

(d)

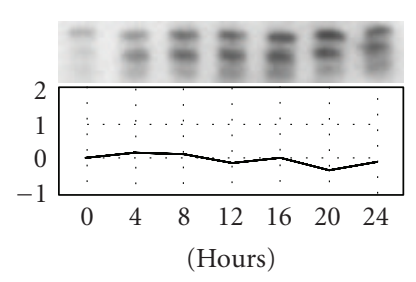

(f)
FIGURE 2: Comparison of results obtained with microarray and protein blot analysis for changes in protein levels during C. neoformans infection. Protein levels at various time points (top panel) relative to the mRNA levels in microarray analysis (bottom panel) are shown for the following genes: (a) $\beta$-actin, (b) Bcl-x, (c) CD47, (d) Bax, (e) Bad, and (f) Bcl-2. The relative scales of the mRNA expression are indicated on the $y$-axis.

the last three time points were similar, yet more distal related to the early gradual changes of gene profiles ( 0 to 12 hours). At these time points (16, 20, 24 hours), the gene profile seems to reach a plateau. The HBMEC is a homogeneous cell culture. The changes in the gene expression profiles are not due to the heterogeneity of cell cultures. The perturbation of gene expression profiles is most likely due to the presence of $C$. neoformans. Overall, the hierarchical clustering analysis of the mRNA levels reveals a sequential change in HBMEC infected with C. neoformans. The overall profile was altered more prominently at the initial stage of the pathogenhost interaction and subsequently persistent expression.

\subsection{Comparison of gene expression profiles from the microarray and protein levels from Western blot analyses}

To determine the validity of results obtained by the microarrayanalysis, some genes were subjected to Western analysis. These genes included $\beta$-actin, $\mathrm{Bcl}-\mathrm{x}, \mathrm{CD} 47, \mathrm{Bax}, \mathrm{Bad}$, and Bcl-2 (see Figure 2). They were chosen for blot analysis because their protein levels were variable and the affinity of commercially available antibodies. For example, $\beta$-actin mRNA and protein levels are maintained in a constant level 


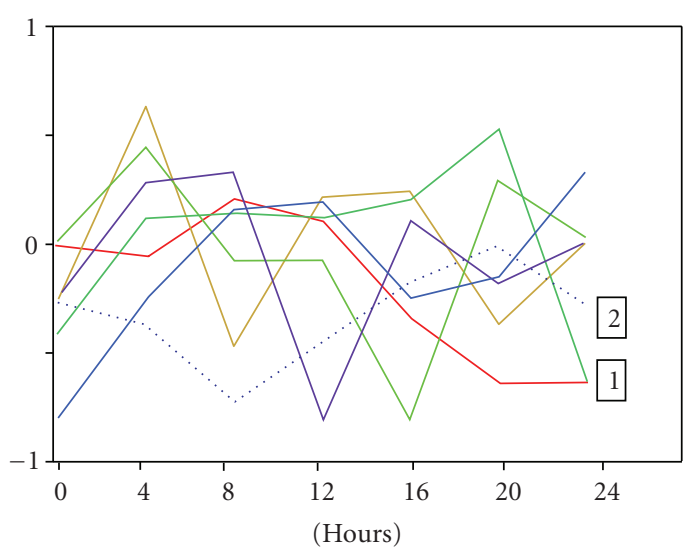

FIgURE 3: PCA of HBMEC profiles after C. neoformans infection; microarray data were analyzed by the GeneSpring software to classify the gene expression patterns. Seven major gene profiles were obtained as shown in different color. The major groups (PCA groups no.1 and no.2) are indicated in the graph, which contain more than $50 \%$ of total analyzed genes.

at all time points, whereas CD47 mRNA and protein level increase to the 8 hour and then decline back to the normal levels. In general, the protein levels of these genes were expressed in varied degrees, and the protein expression profiles were comparable between microarray and protein analyses (see Figure 2). The results validated the competency of the microarray analysis for detection of changes in gene expression in HBMEC during C. neoformans infection.

\subsection{Principal component analysis (PCA) of HBMEC gene profiles during $C$. neoformans infection}

In order to obtain a global grouping of gene profiles, we examined the PCA grouping of mRNA from HBMEC. PCA was a useful linear approach for obtaining a simplified visualization of entire datasets, without losing experimental information (variance). PCA allowed the dimension of complex data to be reduced and the most relevant features of a given dataset (transcriptome) to be highlighted. It was useful for our kinetic studies because it was possible to describe trends that were, otherwise, irretrievably by a direct examination of the entire dataset. The gene fluctuation could be classified into 7 major groups. The major group (PCA group 1) contained 4155 genes (33.08\% of total analyzed genes) and the PCA group 2 contained 3129 genes $(24.92 \%$ of total analyzed genes) (see Figure 3). Thus, these two groups represented more than $50 \%$ of HBMEC mRNAs. The gene profile of PCA group 1 showed a very slightly increase at 8 and 12 hours, and then a rapid decline. On the other hand, the PCA group 2 showed a gradual decline till 8 hours and then bounced back to near the original levels. These two profiles were nearly a mirror image.The results suggested that HBMEC altered its mRNA levels or adjusted its physiological status in response to the pathogen invasion.

\subsection{Gene expression profiles at different time points in HBMEC analyzed by the self-organizing maps (SOM)}

The interpretation of system complexity is the most challenging task of biology in this century. The analysis of complexity in biological systems might start from a simplified representation of static gene networks and then move to an increasingly well-defined and integrated description of biological phenomena, bearing in mind that only dynamic networks will explain reality adequately. An example of a nonhierarchical clustering method is SOM. As SOM solves difficult highdimensional and nonlinear problems. Using this algorithm, gene expression data are transformed into vectors or coordinates in an $n$-dimensional space, where $n$ equals the number of variables or time points. Gene profiles of HBMEC postinfection at 7 time points $(0,4,8,12,16,20,24$ hours) were analyzed by SOM $(7 \times 6)$ (see Figure 4$)$. Upper bound (4.0), normal (1.0), and lower bound (0.0) were shown. The dynamic of gene expression during pathogen-host interaction can be clearly observed (see Figure 4). The use of SOM for grouping of different profiles greatly facilitates further analysis.

\subsection{Persistently upregulated and down-regulated genes during $C$. neoformans infection}

Despites significant fluctuation of gene expression profiles in most HBMEC genes in response to C. neoformans infection, there were some genes that were upregulated or downregulated persistently during 24 hours period. One example of the upregulated genes was the cytochrome $\mathrm{P} 450$ gene which was continuously upregulated through the course of infection. A similar gene profile could be found and grouped, for example, genes that had a correlation at least 0.95 to the expression profile of cytochrome $\mathrm{P} 450$ were defined as upregulated genes (see Figure 5(a)). In the same manner, downregulated genes were grouped, in which genes that had a correlation at least 0.95 to the expression profile of troponin I type 3 gene TNNI3 (see Figure 5(b)).

\subsection{Induced fluctuation of interferons and MHC group genes during $C$. neoformans infection}

We have performed the hierarchical and gene tree analyses to test whether the interferon genes and MHC-related genes were fluctuation during $C$. neoformans infection. The group of interferon-related genes (66 genes) was clustered based on their expression level (see Figure 6). Most genes were fluctuated during the course of infection, suggesting interferon played a role in the innate immune response to $C$. neoformans. Many infectious microbes induced expression of chemokines and adhesion molecules in human endothelial cells. Previous studies of the expression of IL-8, INF- $\gamma$ inducible protein-10 (IP-10), MCP-1, and the leukocyte ligand ICAM-1 in primary HUVEC revealed that C. neoformans had the ability to interfere with inflammatory signaling in human endothelial cells, and suggested that $C$. neoformans might induce leukocyte activation and trafficking in 


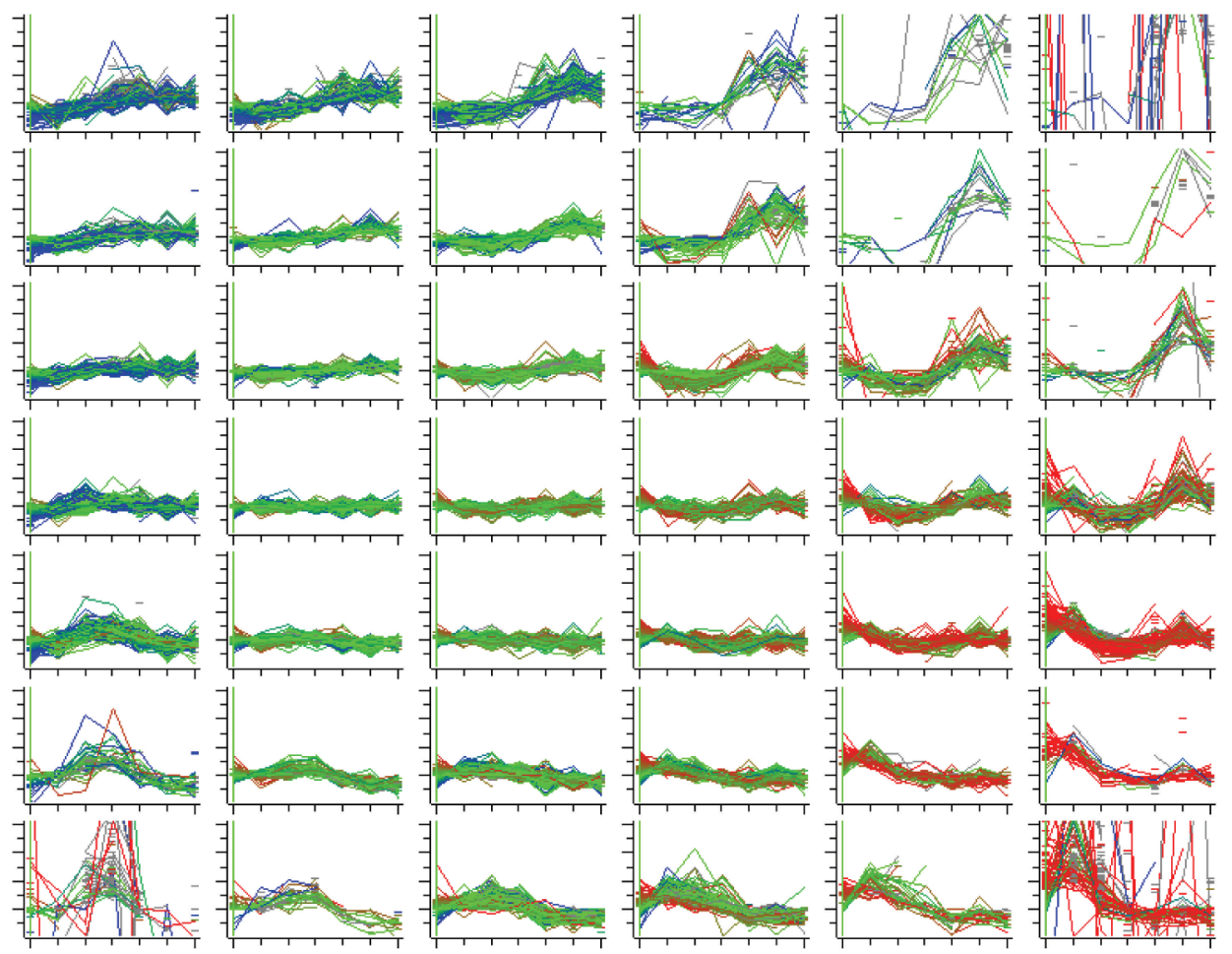

FIGURE 4: Infectomic profiles of $C$. neoformans-infected HBEMC using SOM analysis. Gene profiles of HBMEC postinfection at 7 time points $(0,4,8,12,16,20,24$ hours) ( $x$-axis) were analyzed by SOM ( 7 x 6$)$. Upper bound (4.0), normal (1.0), and lower bound ( 0.0$)$ were shown ( $y$-axis).

the infected host (HUVEC) [5, 6]. Our results showed that IL-1 increased slightly, while MCP-1 did not change significantly. No changes in IP-10 and ICAM-1 expressions were observed in this chip. The TNF- $\alpha$ profile was also increased, though quite late, during the infection. Similarly, the expression of a group of MHC genes including 29 of those in MHC II class was fluctuated (see Figure 7). Although some genes were listed but not expressed, our results suggested that endothelial cells contributed to the host immune response to $C$. neoformans infection. Taken together, HBMEC is not a professional immune cell; however, the fluctuation in the expression of interferons and MHC genes suggests that the innate immune systems are activated during the pathogen invasion.

\section{DISCUSSION}

The current work demonstrates the use of cDNA microarraybased infectomic approach to characterize transcriptomes in HBMEC infected with the meningitic pathogen $C$. neoformans. Like most of meningitic pathogens, the penetration across the $\mathrm{BBB}$ that is constituted by BMEC is required for the pathogenesis of the CNS infection caused by $C$. neoformans $[6,14]$. Our study represents the first investigation of the holistic transcriptional response of the in vitro $\mathrm{BBB}$ cell system in response to $C$. neoformans infection. A total of 12,559 human genes have been analyzed in this study, and distinct alterations in HBMEC gene expression have been observed at $4,8,12,16,20$, and 24 hours of infection. Significant changes in the transcriptional infectomes were ob- served in HBMEC infected with $C$. neoformans. This approach was used to define the infectomic profiles of HBMEC infected with $C$. neoformans for a series of time points, thus, evaluating the dynamic changes in gene expression profiles and inflammatory factors in the host response to this fungal pathogen. The data were analyzed with several clustering analyses. In a hierarchical clustering analysis, the gene expression patterns at different time points displayed a progressive change of gene profiles. The first three incubated time points were clustered, and showed more drastic changes at initial engagement between $C$. neoformans and HBMEC ( 4 hours). The gene partners of the latter time points (16, 20, 24 hours) were clustered together, indicating the alternations reached to a plateau. Microarray analysis has been used for monitoring host gene expression profiles from other pathogen invasion studies, such as HIV-1. Many of the results were to compare the data pre- and post- infections. Our studies revealed that pathogen invasions are multifaceted and dynamics. A time point study is necessary to monitor the alterations. In a nonhierarchical analysis SOM, the twodimensional space map displayed different expression profiles form 12,559 genes into 42 groups. One interesting finding defined by PCA is that one group of genes with graduate reduction in expression ( $\sim 33 \%$ of total genes) accompanied with another group of genes showing gradually increased expression profile ( $\sim 25 \%$ of total genes). The alternative changes in the two major groups (1 and 2) are shown as a mirror image (see Figure 3 ). The biological mechanism and significance of the expression profile changes in 


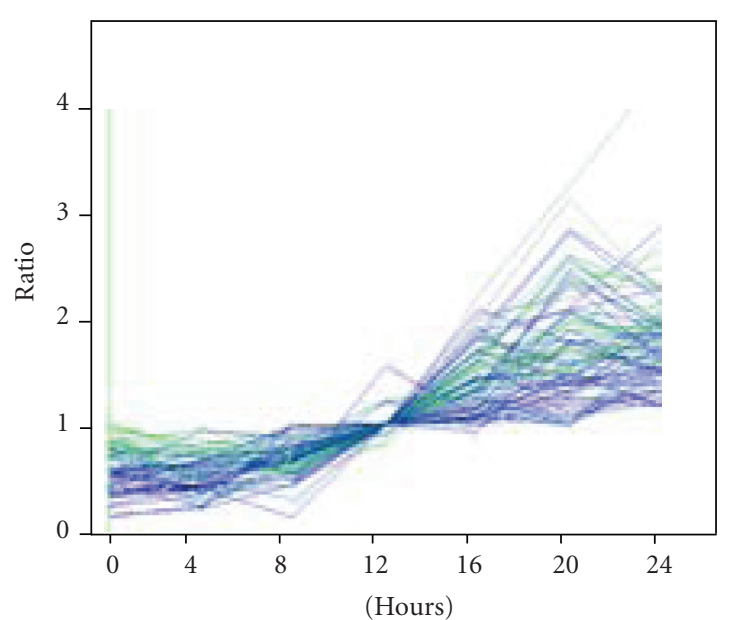

(a)

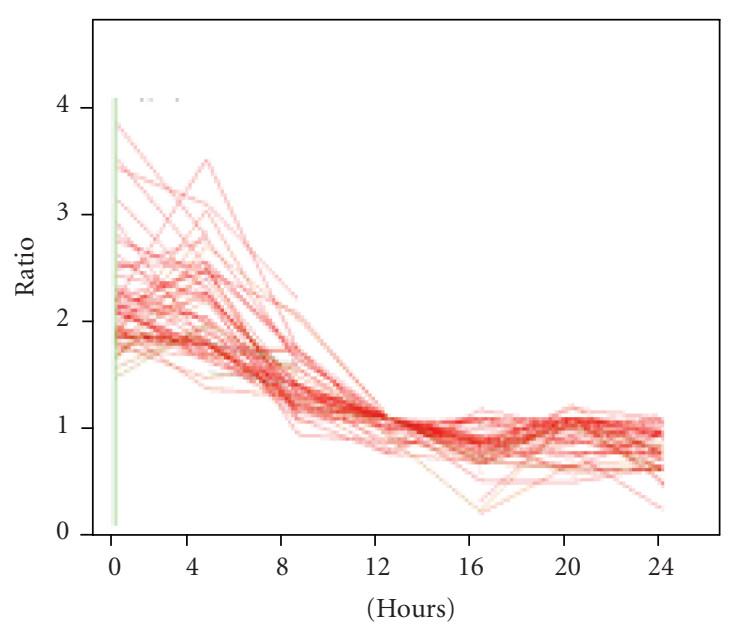

(b)

Figure 5: The upregulated and downregulated gene profiles of HBMEC during C. neoformans infection. (a) Upregulated genes (left chart): cytochrome P450 gene was continuously upregulated during the course of infection. Genes that have correlation at least 0.95 to the expression profile of cytochrome P450 are grouped and defined as upregulated genes. (b) Downregulated genes (right chart): TNNI3 gene was continuously downregulated during the course of infection. Genes that have correlation at least 0.95 to the expression profile of TNNI3 are grouped and defined as downregulated genes.

the pathogenesis of $C$. neoformans infection remain to be defined.

Most interestingly, the group of interferon-related genes (66 genes) was clustered based on their expression level. The expression of most genes fluctuated during the course of infection, suggesting that interferons play a role in the innate immune response to the pathogen. The expression of 29 MHC II class also fluctuated. Though some genes were listed but not expressed, the results suggested that endothelial cells contributed to the host immune response to $C$. neoformans infection. Many infectious microorganisms induced expression of chemokines and adhesion molecules in human endothelial cells. Studies of the gene expression in primary HU-

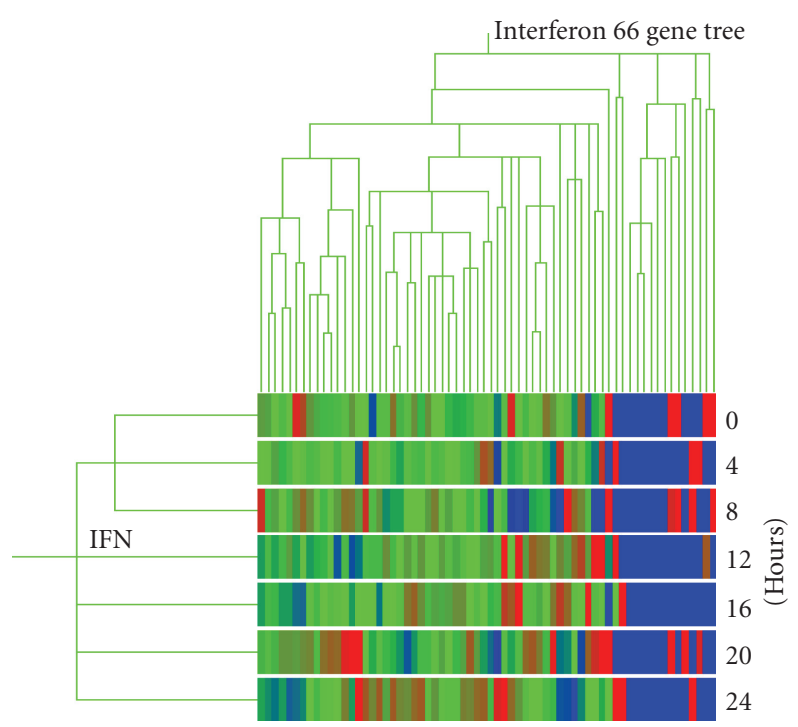

Figure 6: Hierarchical cluster of interferon 66 gene tree. Different expression of 66 interferon-related genes were analyzed by Gene Tree program. The expression of more than two thirds (red and blue) was changed, and about one third of the genes were not expressed (green).

VEC revealed that $C$. neoformans had the ability to interfere with inflammatory signaling in human endothelial cells, and suggested that $C$. neoformans may later induce leukocyte activation and trafficking in the infected host (HUVEC) $[15,16]$. Our results also showed that expression of interferon-related genes and MHC group genes are changed during $C$. neoformans infection. Collectively, the fluctuation in the expression of innate-related genes suggests that $C$. neoformans, like other microbial pathogens, is able to induce expression of chemokines and other innate genes.

The BBB has been considered an immunologically inactive organ as there are few antigen-presenting cells present in the CNS and the presence of the tight junction was thought to prevent the entry of immune cells from the peripheral circulations into the CNS [8]. However, increasing number of studies indicate that the endothelium of the BBB constitutes a dynamic and immunoactive interface between the blood and the CNS that can be modulated by endogenous factors such as bradykinin and cytokines, as well as exogenous factors including meningitic pathogens and their products [17]. Alterations in the BBB function are critical for the development of CNS infection including cryptococcal meningoencephalitis. It has been learnt about the immune response to cryptococcal infection from both in vitro and in vivo studies with the focus on immune cells $[6,18]$. The immune response to $C$. neoformans infection was observed as one of the most interesting changes.

\section{CONCLUSION}

In summary, the current infectomic studies with a genome survey of $C$. neoformans-induced host response in HBMEC provide global information for the pathogenesis of the CNS infection caused by this fungus. Similar conclusions 


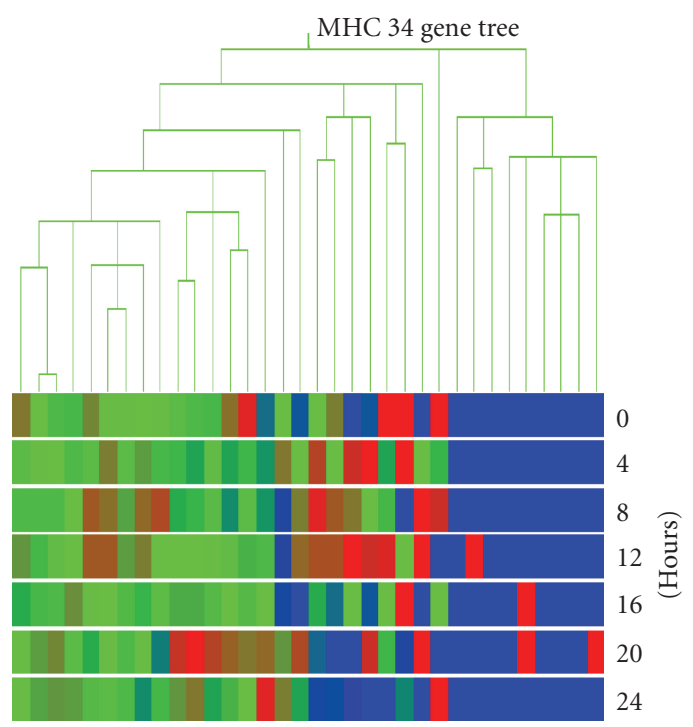

FIGURE 7: Gene tree of MHC genes fluctuated during C. neoformans infection: expression profiles changes in HBMEC are observed. A subgroup of the profile with MHC class genes was selected and subjected to Gene Tree analysis using GeneSpring ${ }^{\mathrm{TM}}$ software. The alternations of gene expression in this group suggested that innate immune contributes to C. neoformans infection.

were reached when analyzing the microarray data with two complementary approaches, PCA and SOM. However, PCA works well in problem spaces that are linearly separable. SOM solves difficult high-dimensional and nonlinear problems. Most importantly, our study demonstrates for the first time that the BBB can actively participate in immune response to cryptococcal infection by regulating the expression of interferons, MHC and cytokines. It suggests that the BBB is not only an impermeable cellular barrier but also constitutes an immunoactive interface between the circulatory system and the CNS and that can be modulated by meningitic pathogens such as $C$. neoformans. Further insight into the role of the $\mathrm{BBB}$ in the pathogenesis of microbial meningitis and immunoregulation of the host defense against meningitic pathogens will gain a global view of the CNS infection and offer exciting prospects for advances in the prevention and therapy of this disease.

\section{ACKNOWLEDGMENTS}

The authors would like to thank Dr. K. J. Kwon-Chung and Dr. Y. Chang for providing C. neoformans strains and intellectual inputs, and Dr. Kwang S. Kim for providing HBMEC. They also thank their colleagues for suggestions, help, and discussion. This work was supported in part by grants-in-aid from the NIH Grant no. RO1 NS047599 (A.Y.J.) and Grant no. RO1 AI40635 (S.H.H.).

\section{REFERENCES}

[1] S.-H. Huang, T. Triche, and A. Jong, "Infectomics: genomics and proteomics of microbial infections," Functional \& Integrative Genomics, vol. 1, no. 6, pp. 331-344, 2002.
[2] T. G. Mitchell and J. R. Perfect, "Cryptococcosis in the era of AIDS-100 years after the discovery of Cryptococcus neoformans," Clinical Microbiology Reviews, vol. 8, no. 4, pp. 515548, 1995.

[3] M. Gottfredsson and J. R. Perfect, "Fungal meningitis," Seminars in Neurology, vol. 20, no. 3, pp. 307-322, 2000.

[4] J. R. Perfect, B. Wong, Y. C. Chang, K. J. Kwon-Chung, and P. R. Williamson, "Cryptococcus neoformans: virulence and host defences," Journal of Medical and Veterinary Mycology, vol. 3, supplement 1, pp. 79-86, 1998.

[5] M. Feldmesser, Y. Kress, P. Novikoff, and A. Casadevall, “Cryptococcus neoformans is a facultative intracellular pathogen in murine pulmonary infection," Infection and Immunity, vol. 68, no. 7, pp. 4225-4237, 2000.

[6] T. Bicanic and T. S. Harrison, "Cryptococcal meningitis," British Medical Bulletin, vol. 72, no. 1, pp. 99-118, 2005.

[7] G. B. Huffnagle and L. K. McNeil, "Dissemination of C. neoformans to the central nervous system: role of chemokines, Th1 immunity and leukocyte recruitment," Journal of NeuroVirology, vol. 5, no. 1, pp. 76-81, 1999.

[8] L. L. Rubin and J. M. Staddon, "The cell biology of the bloodbrain barrier," Annual Review of Neuroscience, vol. 22, pp. 1128, 1999.

[9] R. D. Broadwell, B. J. Baker-Cairns, P. M. Friden, C. Oliver, and J. C. Villegas, "Transcytosis of protein through the mammalian cerebral epithelium and endothelium. III. Receptor-mediated transcytosis through the blood-brain barrier of blood-borne transferrin and antibody against the transferrin receptor," Experimental Neurology, vol. 142, no. 1, pp. 47-65, 1996.

[10] S.-H. Huang and A. Jong, "Cellular mechanisms of microbial proteins contributing to invasion of the blood-brain barrier," Cellular Microbiology, vol. 3, no. 5, pp. 277-287, 2001.

[11] S. H. M. Chen, M. F. Stins, S.-H. Huang, et al., "Crytptococcus neoformans induces alterations in cytoskeleton of human rain microvascular endothelial cells," Journal of Medical Microbiology, vol. 52, pp. 961-970, 2003.

[12] Y. C. Chang, A. Jong, S.-H. Huang, P. Zerfas, and K.J. KwonChung, "CPS1, a homolog of the Streptococcus pneumoniae type 3 polysaccharide synthase gene, is important for the pathobiology of Cryptococcus neoformans," Infection and Immunity, vol. 74, no. 7, pp. 3930-3938, 2006.

[13] A. Jong, M. F. Stins, S.-H. Huang, S. H. M. Chen, and K. S. Kim, "Traversal of Candida albicansacross human blood-brain barrier in vitro," Infection and Immunity, vol. 69, no. 7, pp. 4536-4544, 2001.

[14] K. E. Black and L. R. Baden, "Fungal infections of the CNS: treatment strategies for the immunocompromised patient," CNS Drugs, vol. 21, no. 4, pp. 293-318, 2007.

[15] T. R. Traynor and G. B. Huffnagle, "Role of chemokines in fungal infections," Medical Mycology, vol. 39, no. 1, pp. 41-50, 2001.

[16] N. Mozaffarian, A. Casadevall, and J. W. Berman, "Inhibition of human endothelial cell chemokine production by the opportunistic fungal pathogen Cryptococcus neoformans," Journal of Immunology, vol. 165, no. 3, pp. 1541-1547, 2000.

[17] A. A. Siddiqui, A. E. Brouwer, V. Wuthiekanun, et al., "IFN- $\gamma$ at the site of infection determines rate of clearance of infection in cryptococcal meningitis," Journal of Immunology, vol. 174, no. 3, pp. 1746-1750, 2005.

[18] J. R. Graybill, “Cryptococcal meningitis," Brazilian Journal of Infectious Diseases, vol. 1, pp. 60-67, 1997. 

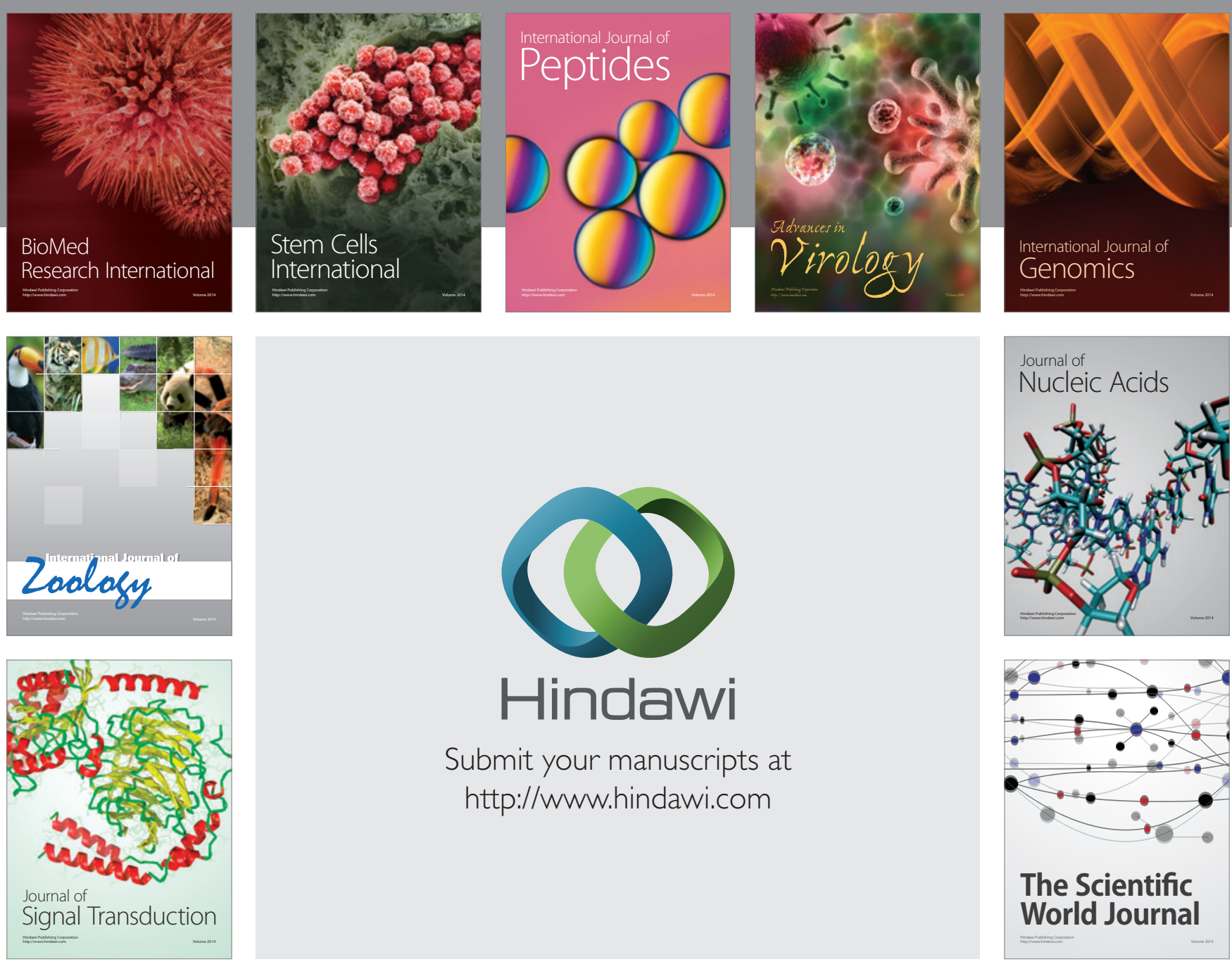

Submit your manuscripts at

http://www.hindawi.com
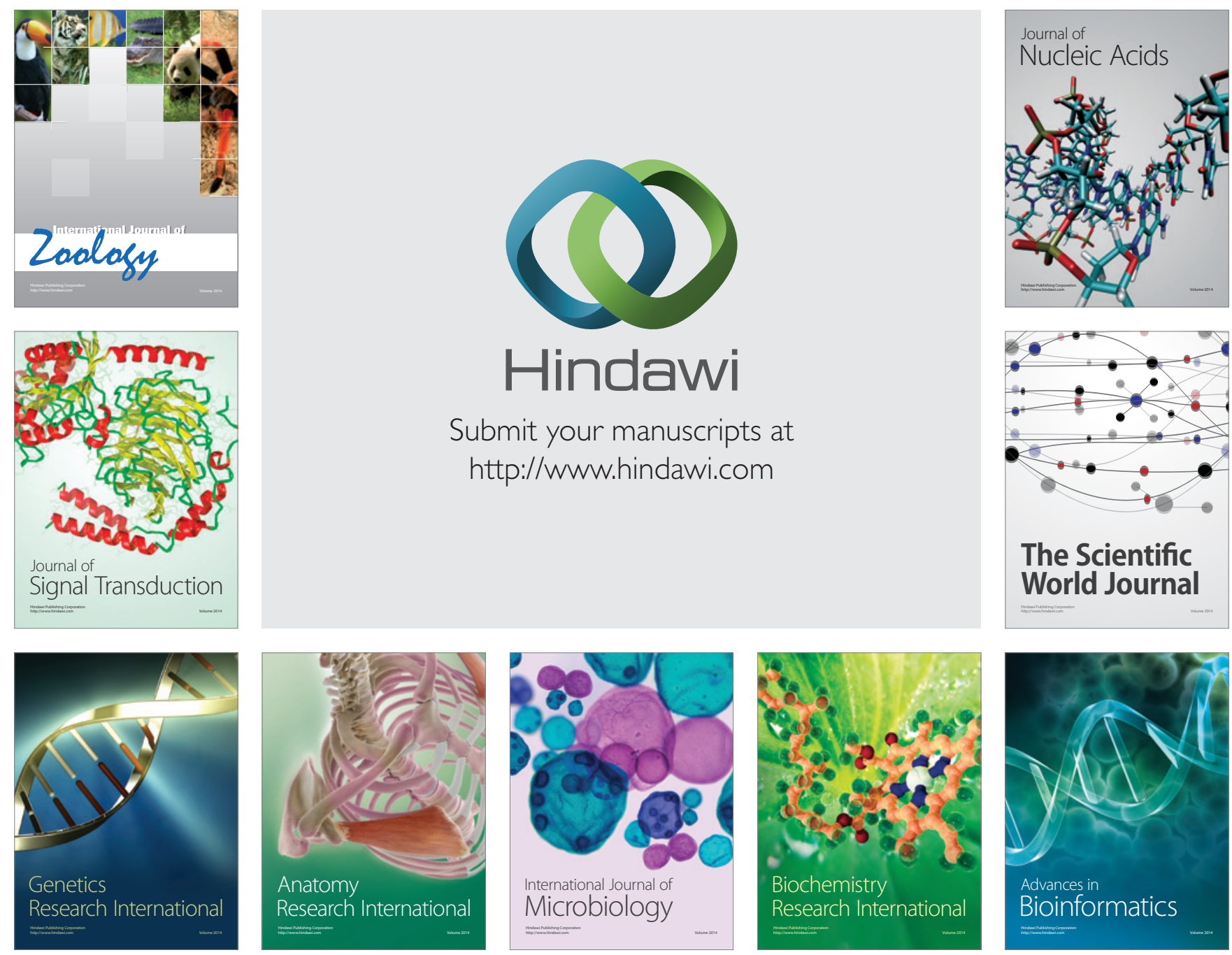

The Scientific World Journal
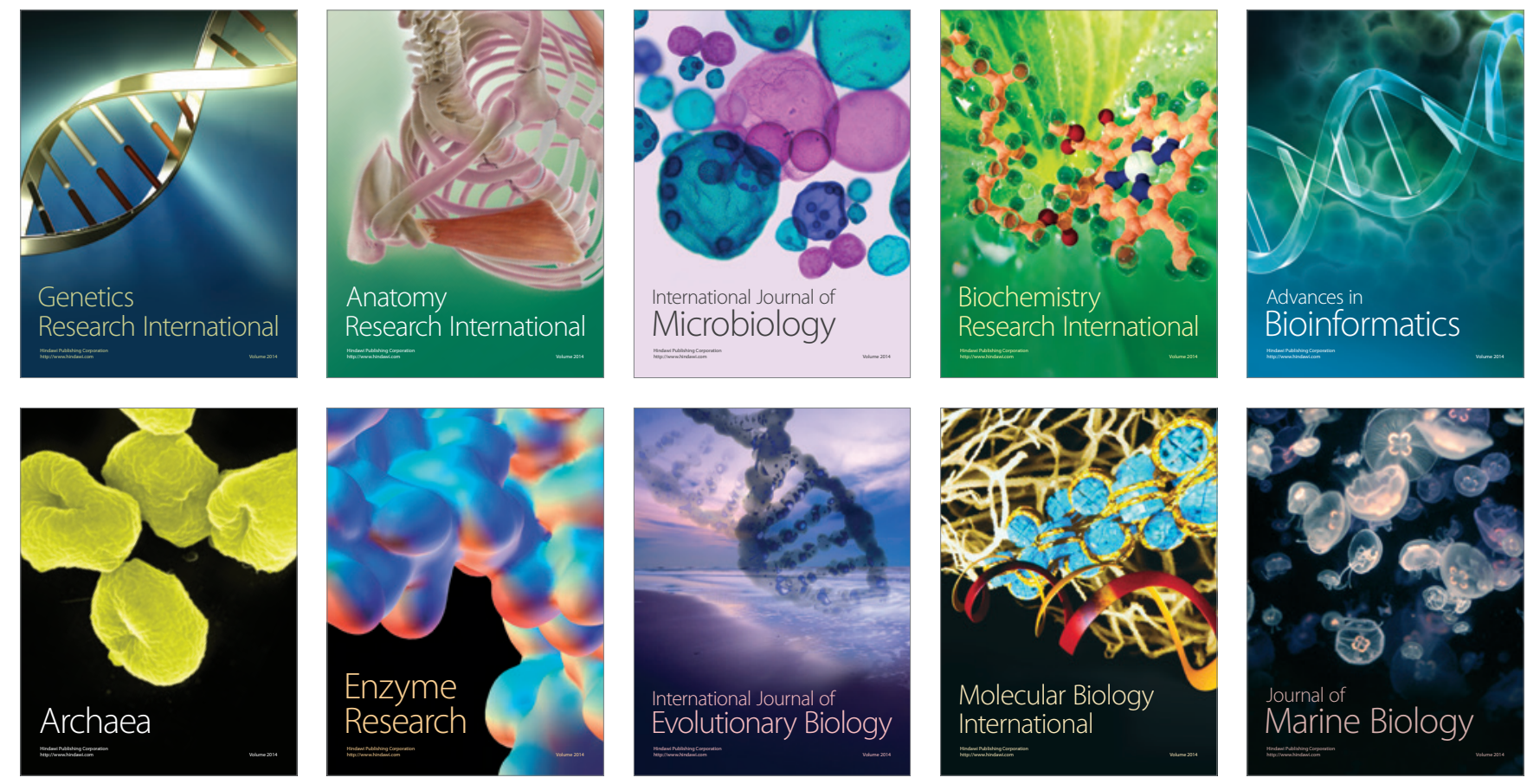